\title{
STUDIES FOR MUON COLLIDER PARAMETERS AT CENTER-OF-MASS ENERGIES OF 10 TEV AND 100 TEV
}

\author{
Bruce J. King, Brookhaven National Laboratory ${ }^{1}$
}

\begin{abstract}
Parameter lists are presented for speculative muon colliders at center-of-mass energies of $10 \mathrm{TeV}$ and $100 \mathrm{TeV}$. The technological advances required to achieve the given parameters are itemized and discussed, and a discussion is given of the design goals and constraints. An important constraint for multi-TeV muon colliders is the need to minimize neutrino radiation from the collider ring.
\end{abstract}

\section{INTRODUCTION}

The main motivation for research and development efforts on muon collider technology is the assertion that affordably priced muon colliders might provide lepton-lepton collisions at much higher center of mass $(\mathrm{CoM})$ energies than is feasible for electron colliders, and perhaps eventually explore the spectrum of elementary particles at mass scales inaccessible even to hadron colliders.

This paper attempts a first look at these assertions through discussion and evaluation of the self-consistent muon collider parameter sets given in table 1 , at CoM energies of $10 \mathrm{TeV}$ and $100 \mathrm{TeV}$. These parameter sets have the purpose of pin-pointing the challenges of very high energy muon colliders and they have not been studied or discussed in detail within the Muon Collider Collaboration (MCC) or elsewhere.

The $10 \mathrm{TeV}$ parameter set was presented previously [1] and the parameter values appear to be internally consistent. In contrast, the $100 \mathrm{TeV}$ parameter set represents work in progress to improve in luminosity and other parameters over the self-consistent $100 \mathrm{TeV}$ parameter set given in reference [1] and it's parameters are not yet fully consistent, as discussed below.

\section{GENERATION OF PARAMETER SETS}

As described previously [1], the parameter sets in table 1 were generated through iterative runs of a stand-alone FORTRAN program, LUMCALC. The parameter sets are calculated from the input values for several input parameters - namely, the CoM energy $\left(\mathrm{E}_{\mathrm{CoM}}\right)$, the collider ring circumference (C) and depth below the Earth's surface (D), the beam momentum spread $(\delta)$ and 6-dimensional invariant emittance $\left(\epsilon_{6 \mathrm{~N}}\right)$, the reference pole-tip magnetic field for the final focus quadrupoles $\left(\mathrm{B}_{4 \sigma}\right)$, and the time until the beams are dumped $\left(t_{D}\right)$ - and from the input of maximum allowable values for several other parameters - namely, the bunch repetition frequency $\left(f_{b}\right)$, the initial number of

\footnotetext{
1 web page: http://pubweb.bnl.gov/people/bking/ email: bking@bnl.gov. This work was performed under the auspices of the U.S. Department of Energy under contract no. DE-AC02-98CH10886.
}

muons per bunch $\left(N_{0}\right)$, the beam-beam tune disruption parameter $(\Delta \nu)$, the beam divergence at the interaction point $\left(\sigma_{\theta}\right)$, the maximum aperture for the final focus quadrupoles $\left(\mathrm{A}_{ \pm 4 \sigma}\right)$, and maximum allowable neutrino radiation where the plane of the collider ring cuts the Earth's surface.

As a preliminary stage of calculation, LUMCALC makes any parameter adjustments that may be required to satisfy the input constraints. These are, in order: 1) reducing $\sigma_{\theta}$ to the limit imposed by $\mathrm{A}_{ \pm 4 \sigma}$ (based on scaling to existing final focus designs at $0.1 \mathrm{TeV}$ and $4 \mathrm{TeV}[1]), 2$ ) reducing $\mathrm{N}_{0}$ to attain an acceptable $\Delta \nu$, and 3) reducing $\mathrm{f}_{\mathrm{b}}$ until the neutrino radiation is acceptable.

\section{DISCUSSION}

The physics motivation for each of the parameter sets in table 1 is discussed in [2]. Briefly, the number of $\mu \mu \rightarrow$ ee events gives a benchmark estimate of the discovery potential for elementary particles at the full CoM energy of the collider, while the production of hypothesized $100 \mathrm{GeV}$ Higgs particles indicates roughly how the colliders would perform in studying physics at this fixed energy scale.

Both parameter sets give exciting luminosities with good potential to explore the physics processes at and below their respective CoM energy scales.

The output luminosity may be derived in terms of the input parameters as:

$$
\begin{aligned}
\mathcal{L}\left[\mathrm{cm}^{-2} \cdot \mathrm{s}^{-1}\right]= & 2.11 \times 10^{33} \times \mathrm{H}_{\mathrm{B}} \times\left(1-\mathrm{e}^{-2 \mathrm{t}_{\mathrm{D}}\left[\gamma \tau_{\mu}\right]}\right) \\
& \times \frac{\mathrm{f}_{\mathrm{b}}\left[\mathrm{s}^{-1}\right]\left(\mathrm{N}_{0}\left[10^{12}\right]\right)^{2}\left(\mathrm{E}_{\mathrm{CoM}}[\mathrm{TeV}]\right)^{3}}{\mathrm{C}[\mathrm{km}]} \\
& \times\left(\frac{\sigma_{\theta}[\mathrm{mr}] \cdot \delta\left[10^{-3}\right]}{\epsilon_{6 \mathrm{~N}}\left[10^{-12}\right]}\right)^{2 / 3}
\end{aligned}
$$

This formula uses the standard MCC assumption[1] that the ratio of transverse to longitudinal emittances can be chosen in the muon cooling channel to maximize the luminosity for a given $\epsilon_{6 \mathrm{~N}}$. The pinch enhancement factor, $\mathrm{H}_{\mathrm{B}}$, is very close to unity (see table 1), and the numerical coefficient in equation 1 includes a geometric correction factor of 0.76 for the non-zero bunch length, $\sigma_{z}=\beta^{*}$ (the "hourglass effect").

In practice, the muon beam power and current are limited so the optimization of parameters actually involves the "specific luminosity"

$$
l \equiv \frac{\mathcal{L}}{f_{b} \times N_{0}} .
$$

Further, the parameter sets at these high energies are always limited by $\Delta \nu$ and it is useful and easy to rewrite 
equations 1 and 2 in the form

$$
l \propto \frac{\Delta \nu}{\beta *},
$$

with no explicit dependence on emittance for a given energy. This is misleading, however, since smaller emittances mean smaller magnet apertures and hence allow the design of lattices with smaller $\beta *$ 's and, in practice, one can almost always gain in specific luminosity by reoptimizing parameter sets with smaller emittances.

The assumed 6-dimensional emittances are factors of $3.5(10 \mathrm{TeV})$ and $50(100 \mathrm{TeV})$ smaller than the value $170 \times 10^{-12} \mathrm{~m}^{3}$ that is normally used in MCC scenarios for first generation muon colliders, in anticipation that the muon cooling channel may eventually be improved through further design optimization, stronger magnets, higher gradient rf cavities and other technological advancements and innovations.

The final focus region presumably presents the most difficult design challenge that is relatively specific to high energy muon colliders. (The muon cooling channel is, of course, a formidable challenge for all muon colliders.) Progressively stronger focusing is required at higher energies to generate the smaller spot sizes necessary to increase the luminosity. The strength of the focusing can be gauged from the overall beam demagnification, defined as $M \equiv \sqrt{\beta_{\max } / \beta^{*}}$. This is a dimensionless parameter that should be closely correlated with fractional tolerances in magnet uniformity, residual chromaticity etc. Hence, it might be prudent to decrease the fractional momentum spread of the beams, $\delta$, to compensate for an increasing $M$. In the absence of final focus designs for these parameter sets the difficulty of the chromatic correction for the final focus has simplistically been assessed by the value of a single parameter: the "chromaticity quality factor" $q \equiv M \delta[3]$.

In the absence of "proof by example" lattice designs, the next generation of proposed linear e+e- colliders (LC's) may well provide the best benchmarks for heuristically assessing the difficulty of the final focus parameters. For the $10 \mathrm{TeV}$ parameter set, each of $\beta *, M$ and $q$ are roughly comparable to those proposed for these LC's. The $100 \mathrm{TeV}$ parameter set necessarily has a much larger $\beta *$ and $M$ but the value of $\delta$ was forced down to $\delta=8 \times 10^{-5}$ to nearly hold steady the value of $q$. Unfortunately, this is clearly inconsistent with the rising energy loss due to beamstrahlung at the ip. Following the lead of the LC's, the next iteration of the $100 \mathrm{TeV}$ parameter set will use colliding flat beams to make the parameter sets fully consistent. The spot size - clearly indicative of vibration and alignment tolerances also falls with energy, but even at $100 \mathrm{TeV}$ it remains an order of magnitude above the spot size in the y coordinate for future LC design parameters.

Neutrino radiation is an extremely serious problem for many-TeV muon colliders and further information and discussion on this can be found in these proceedings [4]. The radiation levels are predicted to rise rapidly with the col- lider energy [4] and beyond CoM energies of a few TeV it may well be necessary to build the colliders at isolated sites where the public would not be exposed to the neutrino radiation disk.

\section{CONCLUSIONS}

Muon colliders from 10 to $100 \mathrm{TeV}$ CoM energies may offer an exciting long-term future to experimental HEP and are not obviously infeasible to this author. However, they clearly introduce some daunting new challenges beyond those common to all muon colliders and the parameter sets in table 1 will require some technological extrapolations.

\section{REFERENCES}

[1] The Muon Collider Collaboration, "Status of Muon Collider Research and Development and Future Plans", to be submitted to Phys. Rev. E.

[2] B.J. King, "Discussion on Muon Collider Parameters at Center of Mass Energies from $0.1 \mathrm{TeV}$ to 100 TeV", 19 June, 1998, Submitted to Proc. Sixth European Particle Accelerator Conference (EPAC'98), Stockholm, Sweden, 22-26 June, 1998. Available at http://pubweb.bnl.gov/people/bking/.

[3] The idea to use this parameter emerged through discussions with Frank Zimmermann and Carol Johnstone .

[4] B.J. King, "Potential Hazards from Neutrino Radiation at Muon Colliders", these proceedings. 
Table 1: Example parameter sets for $10 \mathrm{TeV}$ and $100 \mathrm{TeV}$ muon colliders. The generation of these parameter sets is discussed in the text. These parameters represent speculation by the author on how muon colliders might evolve with energy. The beam parameters at the interaction point are defined to be equal in the horizontal (x) and vertical (y) transverse coordinates.

\begin{tabular}{|c|c|c|}
\hline center of mass energy, $\mathrm{E}_{\mathrm{CoM}}$ & $10 \mathrm{TeV}$ & $100 \mathrm{TeV}$ \\
\hline \multicolumn{3}{|l|}{ collider physics parameters: } \\
\hline luminosity, $\mathcal{L}\left[\mathrm{cm}^{-2} \cdot \mathrm{s}^{-1}\right]$ & $1.0 \times 10^{36}$ & $3.1 \times 10^{37}$ \\
\hline $\int \mathcal{L} \mathrm{dt}\left[\mathrm{fb}^{-1} / \operatorname{det} /\right.$ year $]$ & 10000 & 310000 \\
\hline No. of $\mu \mu \rightarrow$ ee events/det/year & 8700 & 2700 \\
\hline No. of $100 \mathrm{GeV}$ SM Higgs/det/year & $1.4 \times 10^{7}$ & $6.5 \times 10^{8}$ \\
\hline fract. CoM energy spread, $\sigma_{\mathrm{E}} / \mathrm{E}\left[10^{-3}\right]$ & 1.0 & 0.08 \\
\hline \multicolumn{3}{|l|}{ collider ring parameters: } \\
\hline circumference, $\mathrm{C}[\mathrm{km}]$ & 15 & 100 \\
\hline ave. bending B field $[\mathrm{T}]$ & 7.0 & 10.5 \\
\hline \multicolumn{3}{|l|}{ beam parameters: } \\
\hline$\left(\mu^{-}\right.$or $) \mu^{+} /$bunch, $\mathrm{N}_{0}\left[10^{12}\right]$ & 2.4 & 2.0 \\
\hline ( $\mu^{-}$or) $\mu^{+}$bunch rep. rate, $\mathrm{f}_{\mathrm{b}}[\mathrm{Hz}]$ & 15 & 10 \\
\hline 6-dim. norm. emittance, $\epsilon_{6 N}\left[10^{-12} \mathrm{~m}^{3}\right]$ & 50 & 3.5 \\
\hline $\mathrm{x}, \mathrm{y}$ emit. (unnorm.) $[\pi . \mu \mathrm{m} . \mathrm{mrad}]$ & 0.55 & 0.0046 \\
\hline $\mathrm{x}, \mathrm{y}$ normalized emit. $[\pi \cdot \mathrm{mm} . \mathrm{mrad}]$ & 26 & 22 \\
\hline fract. mom. spread, $\delta\left[10^{-3}\right]$ & 1.4 & 0.084 \\
\hline relativistic $\gamma$ factor, $\mathrm{E}_{\mu} / \mathrm{m}_{\mu}$ & 47322 & 473220 \\
\hline ave. current $[\mathrm{mA}]$ & 24 & 7.9 \\
\hline beam power [MW] & 58 & 320 \\
\hline decay power into magnet liner $[\mathrm{kW} / \mathrm{m}]$ & 1.4 & 2.4 \\
\hline time to beam dump, $\mathrm{t}_{\mathrm{D}}\left[\gamma \tau_{\mu}\right]$ & no dump & 0.5 \\
\hline effective turns/bunch & 1039 & 985 \\
\hline \multicolumn{3}{|l|}{ interaction point parameters: } \\
\hline spot size, $\sigma_{x}=\sigma_{y}[\mathrm{~nm}]$ & 780 & 93 \\
\hline bunch length, $\sigma_{z}[\mathrm{~mm}]$ & 1.1 & 0.185 \\
\hline$\beta^{*}[\mathrm{~mm}]$ & 1.1 & 0.185 \\
\hline ang. divergence, $\sigma_{\theta}[\mathrm{mrad}]$ & 0.71 & 0.5 \\
\hline beam-beam tune disruption parameter, $\Delta \nu$ & 0.100 & 0.100 \\
\hline pinch enhancement factor, $\mathrm{H}_{\mathrm{B}}$ & 1.108 & 1.130 \\
\hline beamstrahlung fract. E loss/collision & $2.3 \times 10^{-7}$ & $6.5 \times 10^{-4}$ \\
\hline \multicolumn{3}{|l|}{ final focus lattice parameters: } \\
\hline max. poletip field of quads., $\mathrm{B}_{4 \sigma}[\mathrm{T}]$ & 15 & 20 \\
\hline max. full aperture of quad., $\mathrm{A}_{ \pm 4 \sigma}[\mathrm{cm}]$ & 20 & 88 \\
\hline$\beta_{\max }[\mathrm{km}]$ & 1100 & 260000 \\
\hline final focus demagnification, $M \equiv \sqrt{\beta_{\max } / \beta^{*}}$ & 31000 & $1.2 \times 10^{6}$ \\
\hline chrom. quality factor, $Q \equiv M \delta$ & 43 & 100 \\
\hline \multicolumn{3}{|l|}{ synchrotron radiation parameters: } \\
\hline syn. E loss/turn $[\mathrm{MeV}]$ & 17 & 25000 \\
\hline syn. rad. power $[\mathrm{MW}]$ & 0.4 & 200 \\
\hline syn. critical E $[\mathrm{keV}]$ & 12 & 1700 \\
\hline \multicolumn{3}{|l|}{ neutrino radiation parameters: } \\
\hline collider reference depth, $\mathrm{D}[\mathrm{m}]$ & 300 & 300 \\
\hline$\nu$ beam distance to surface $[\mathrm{km}]$ & 62 & 62 \\
\hline at surface $[\mathrm{m}]$ & 1.3 & 0.13 \\
\hline str. sect. length for $10 \mathrm{x}$ ave. rad., $\mathrm{L}_{\mathrm{x} 10}[\mathrm{~m}]$ & 1.0 & 2.4 \\
\hline ave. rad. dose in plane $[\mathrm{mSv} / \mathrm{yr}]$ & 0.66 & 12.6 \\
\hline
\end{tabular}

